\title{
COVID-19, Immuno-oncology and Cardiovascular Disease: Viewpoint from the Intersection
}

\author{
Hasan K. Siddiqi ${ }^{1}$ • Tomas G. Neilan ${ }^{2}$ \\ Published online: 4 May 2020 \\ (C) Springer Science+Business Media, LLC, part of Springer Nature 2020
}

The coronavirus disease of 2019 (COVID-19) pandemic continues to ravage populations across the world, with more than 3 million confirmed cases and 200,000 lives lost. Given the rapid spread of disease, the medical community is looking to pre-existing medications, paradigms, and models to better understand and treat COVID-19, necessitating multi-disciplinary expert input to explain the myriad of findings in COVID-19, as well as help formulate novel and effective therapies.

Cardiac injury is commonly seen in patients with COVID19 , and is associated with worse prognosis and mortality [1]. The exact mechanisms of cardiac injury are unknown, but some hypothesized mechanisms include direct viral myocardial injury, microvascular injury, stress cardiomyopathy, myocardial supply-demand mismatch, and/or systemic hyperinflammation resulting in cardiac injury [2]. Published reports of COVID-19 cohorts demonstrate the presence of significant inflammation, with elevated interleukin (IL)-6, high sensitivity C-reactive protein, D-dimer, ferritin, and erythrocyte sedimentation rate. Therefore, there is a significant likelihood that a hyperinflammatory state akin to cytokine release syndrome (CRS) plays an important role in the pathogenesis and prognosis of advanced COVID-19, including its cardiotoxicity.

Cancer immune therapy may provide key insights into the pathobiology of the hyperinflammatory phase in COVID-19 and its effects on the cardiovascular system. Two key contemporary immune therapies are immune checkpoint inhibitors (ICI) and chimeric antigen receptor T cells (CAR-T). With

Tomas G. Neilan

TNEILAN@mgh.harvard.edu

1 Divisions of Cardiovascular and Preventive Medicine, Department of Medicine, Brigham and Women's Hospital, Harvard Medical School, Boston, MA, USA

2 Cardio-Oncology Program, Division of Cardiology, Department of Medicine, Massachusetts General Hospital, Harvard Medical School, Boston, MA, USA
ICIs, monoclonal antibodies block various inhibitory checkpoints (such as PD-1, PD-L1, CTLA-4) in the host's immune system, rendering the immune system able to recognize and more effectively attack tumor cells. In CAR-T therapy, T cells are engineered to express chimeric tumor-associated antigen receptors which recognize tumor antigens and initiate the host's immune response. Although these approaches have provided remarkable success in treating previously aggressive cancers, they may be associated with cardiac injury and cardiovascular events [3]. The mechanisms of cardiotoxicity in ICI and CAR-T therapy are distinct but poorly understood. In ICI cardiotoxicity, blockade of intrinsic checkpoints by antibody administration leads to immune cell-mediated myocarditis, which is associated with significant morbidity and mortality. [4] Cardiotoxicity associated with CAR-T cells is related to CRS, a phenomenon marked by an exuberant release of inflammatory cytokines, with IL-6 thought to be an important mediator of this response [5]. Primary treatment for these immune therapy-related cardiotoxicities is different. For ICI myocarditis, the front-line therapy generally involves broad immunosuppression. For CAR-T-related toxicity, therapy is aimed at reducing the inflammatory milieu and limit organ dysfunction. As such, in severe CRS, IL-6 inhibitors are now a mainstay of therapy with reasonable outcomes.

Given the emerging picture of COVID-19 leading to a hyperinflammatory state with resulting cardiotoxicity, there may be important lessons to learn and apply from our immune therapy experience (Fig. 1). With COVID-19, early data suggest a T cell exhaustion with increased expression of PD-1 and PD-L1. In this setting, blockade of these critical pathways with ICIs may be harmful. Rather, supporting the immune response with a CTLA-4 agonist may be helpful. There has been a hesitance to employ immunomodulators in COVID-19 for fear of potentiating viral replication and reducing host immune viral clearance. However, there is likely a role for immune-modulatory therapy in severe COVID-19, where a dysregulated host immune response is responsible for the capillary permeability and multi-system dysfunction seen in 


\section{Putative mechanisms of cardiotoxicity in COVID-19 and relationship to contemporary immune therapies}

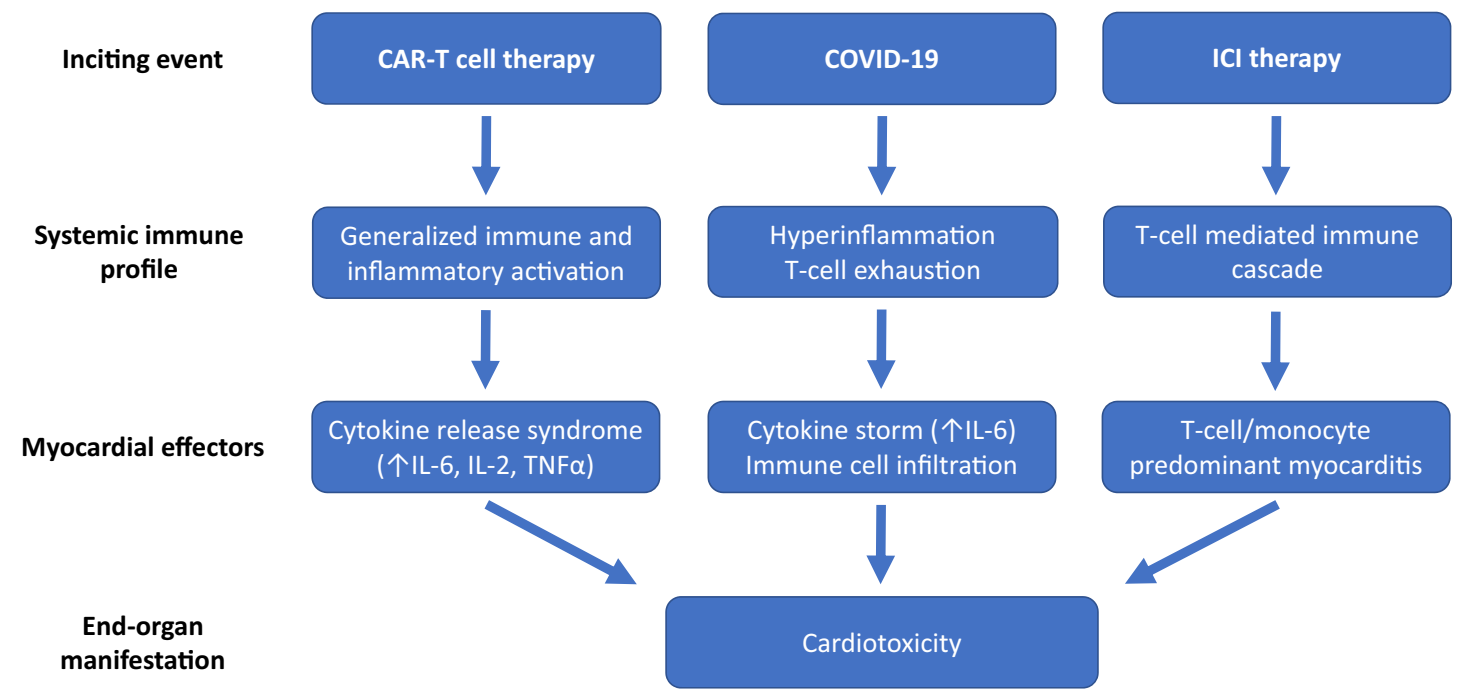

Fig. 1 Putative mechanisms of cardiotoxicity in COVID-19 and relationship to contemporary immune therapies

critically ill patients. In advanced disease, the use of general immunomodulators such as corticosteroids, along with intravenous immunoglobulin, may provide for reduction in the inflammatory environment and rescue vital organs, akin to that seen in ICI myocarditis. More directly, with a key role of IL-6 in COVID-19 hyperinflammation, utilizing IL-6 inhibitors may lead to interruption of the cytokine storm, similar to that with CAR-T myocardial injury. In addition, and beyond the focus of this report, the long-term sequelae of this hyperinflammation to the cardiovascular system are unknown, both with immune therapies and in COVID-19. Indeed, it has been posited that these immune injuries may be risk factors for the development of future cardiovascular disease through fibrosis or accelerated atherosclerosis, among other mechanisms. This identifies a critical need for long-term study of COVID-19 patients to understand cardiovascular sequelae effects of this disease.

In summary, a significant part of the cardiovascular sequelae of COVID-19 is likely due to the exuberant immune activation. There are important parallels to be drawn between this stage of COVID-19 with the cardiac injury seen with both CAR-T cell and ICI therapies, where dysregulation of the immune and inflammatory response can lead to cardiovascular events. Use of immunomodulators in COVID-19 may follow the relative success of such therapies to treat the toxicities of CAR-T and should be heavily considered in cases of patient decline and emerging shock. Only with the application of such inter-disciplinary investigation and therapeutic development can there be an effective and urgent treatment of the global COVID-19 pandemic that threatens millions of lives globally.

\section{References}

1. Shi, S., et al. (2020). Association of cardiac injury with mortality in hospitalized patients with COVID-19 in Wuhan, China. JAMA Cardiology, e200950. https://doi.org/10.1001/jamacardio.2020. 0950.

2. Atri, D., Siddiqi, H. K., Lang, J., et al. (2020). COVID-19 for the cardiologist: a current review of the virology, clinical epidemiology, cardiac and other clinical manifestations and potential therapeutic strategies. JACC: Basic to Translational Science. https://doi.org/10. 1016/j.jacbts.2020.04.002.

3. Zhang, L., Awadalla, M., Mahmood, S. S., et al. (2020). Cardiovascular magnetic resonance in immune checkpoint inhibitor-associated myocarditis [published online ahead of print, 2020 Feb 29]. European Heart Journal, ehaa051. https://doi.org/ 10.1093/eurheartj/ehaa051.

4. Mahmood, S. S., Fradley, M. G., Cohen, J. V., et al. (2018). Myocarditis in patients treated with immune checkpoint inhibitors. Journal of the American College of Cardiology, 71(16), 1755-1764.

5. Alvi, R. M., Frigault, M. J., Fradley, M. G., et al. (2019). Cardiovascular events among adults treated with chimeric antigen receptor T-cells (CAR-T). Journal of the American College of Cardiology, 74(25), 3099-3108. https://doi.org/10.1016/j.jacc. 2019.10.038.

Publisher's Note Springer Nature remains neutral with regard to jurisdictional claims in published maps and institutional affiliations. 